\title{
LA COMUNICAZIONE E GLI USI DELLA LINGUA ITALIANA DI OGGI
}

\author{
Carolina Massi Albanese \\ Universidade Federal do Paraná \\ RIASSUNTO
}

La nostra indagine offre uno sbozzo della situazione attuale della lingua italiana. Sono stati considerati, come dati centrali per l'analisi, la competenza del parlante, la narrativa ed i linguaggi settoriali giornalistico e pubblicitario.

'Le parole figliano parole' è una splendida immagine ripresa da un verso di un poeta siciliano vivente, Ignazio, Butitta. Una lingua è morta, dice Butitta in siciliano, "Quannu i paroli non figghianu paroli"', cioè quando dalle parole di quella lingua non nascono più altre parole.

Negli ultimi anni la derivazione si è sempre più fatta strada, sia per creare parole nuove che designano qualche caratteristica della vita moderna:
benzinaio, benzinaro $=$ persona addetta a un distri- butore di benzina
magliaro $=$ venditore ambulante di tessuti ragazzeria $=$ negozio di abbigliamento sportivo $\mathrm{e}$ varie per ragazzi,

sia per la estensione di espressioni appartenenti al campo della burocrazia, della politica, della tecnica: futuribile, investimento, incremento, programmazione, direzionale, direzionare, dirigenziale, istanza, istanzare, municipalizzare, revisionare, ecc.

Tra i morfemi modificanti derivativi, prevalgono gli usi estensivi di - ese e -oso.

Il suffisso -ese, terminazione derivata dalla lingua latina ( -ense è forma dotta: forense), ravvisato in termini

1 DEVo' O, Glacomo. Civiltá di parole. Firenze, Sansonl, 1965. p.134. 
della cultura medievale: cortese $=$ che è della corte; borghese = abitante del borgo; forese = di fuori città, campagnolo, si usa normalmente per segnalare la nazionalità o la cittadinanza: (Portogallo) - portoghese, (Inghilterra) inglese, (Calabria) - calabrese, 'Abruzzo) - abruzzese, (Milano) - milanese. Recentemente è stato ripreso per indicare modi di parlare propri di gruppi sociali o legati a certe circostanze particolari: ${ }^{2}$

sindacalese $=$ linguaggio tipico dei sindacalisti;

sinistrese $=$ linguaggio tipico di alcuni politicanti della sinistra;

telefonese $=$ linguaggio allusivo per eludere le intercettazioni telefoniche.

Il sufisso -oso, pure di derivazione latina, è quasi sempre applicato a nomi (anticamente anche in aggettivi: robustoso, come nel Cantico di Frate Sole di San Francesco) ed indica "pieno di, dotato di": ozioso, noioso, spazioso. La pubblicità da pochi anni ha lanciato forme come: comodoso, scattoso... Basta riportarsi alla campagna per un automobile prodotta dalla FIAT, la "Uno":

è comodosa

è sciccosa

è risparmiosa

è scattosa

Tutto il resto è relativo!

Oggigiorno si mira più che mai alla scelta di parole motivate, create via via che se ne presenti la necessità. Si nota cosi la tendenza a coniare:

$$
\begin{aligned}
& \text { da nomi }\left\{\begin{array}{c}
\text { altri nomi in -azione } \\
\text { (struttura-strutturazione) } \\
\text { aggettivo in -ale } \\
\text { (decisione-decisionale) } \\
\text { verbi in -izzare } \\
\text { (atomo-atomizzare) }
\end{array}\right. \\
& \text { da aggettivi }\left\{\begin{array}{c}
\text { nomi in -ismo } \\
\text { (plurale-pluralismo) } \\
\text { altri aggettivi in -istico } \\
\text { (plurale-pluralistico), }
\end{array}\right.
\end{aligned}
$$

e vengono fuori espressioni con i seguenti neologismi:

2 Sabatint. Francesco. Pratica, analisi e storia della lingua italiana. Torino, Inttes, 1981. p.490. 
"Si sviluppa il massimalismo anarcoide dei gruppuscoli";

"Questa è la sconfitta dello spontaneismo e dell'assem. blearismo dei lavoratori"3.

Molto diffuso il morfema modificante -ata che colora per lo meno sei significati:

$$
N+\text {-ata } \begin{cases}N_{1} & \text { cucchiaio - cucchiaiata } \\ N_{2} & \text { arco - arcata } \\ N_{3} & \text { mascalzone - mascalzonata } \\ N_{4} & \text { bastone - bastonata } \\ N_{5} & \text { spaghetti - spaghettata } \\ N_{0} & \text { telefono - telefonata }\end{cases}
$$

ossia:

1) 'quanto può essere contenuto in' cucchiaiata, forchettata, bracciata;

2) 'una serie di' scalinata, arcata, graticciata;

3) 'atteggiamento sconsiderato o privo di decoro' ragazzata, pagliacciata, mascalzonata;

4) 'colpo dato con' bastonata, pedata, schioccata;

5) 'azione compiuta in allegra compagnia' bicchierata, passeggiata, spaghettata;

6) 'azione compiuta con' telefonata, pennellata, spazzolata.

Parole straniere, entrate nell'uso comune, si uniscono a suffissi e/o desinenze italiani dopo aver subito un adattamento sbrigativo. Alcuni esempi:

shock - scioccare, scioccante

snob snobbare, snobbato

stress - - stressato, stressante

manager — manageriale

scout - scautismo

Un altro procedimento attinente alla formazione delle parole è quello della composizione. La sintesi, il bisogno di Jrevità è veramente l'anima del nostro secolo per cui si hanno fusioni, o meglio, veri e propri tagli che rendono le parole più corte senza modificarne il significato, quindi, senza formare nuove parole. Sono le abbreviazioni e le sigle: si preferisce 'cine' a 'cinema', 'bici' a 'bicicletta', 'auto' a 'automobile', 'sub' a 'subacqueo', 'tivì' a 'televisione', ecc.

La 'Fabbrica Italiana Automobile Torino' diventa la FIAT; l'Istituto per la Ricostruzione Industriale' diventa 1'IRI e si conia persino il verbo 'irizzare' = sottoporre un'azienda al controllo dell'IRI. Le 'Radio Audizioni Italiane' diventano la RAI e così via.

3 DLATTO \& MORTARA. Messaggio e comunicaziono. Torino, Laescher, 1977. p.137. 
Nella lingua italiana si vanno sviluppando coniazioni di parole con semplice accostamento di termini non uniti da funzionali:

gente bene, caso limite, monte premi, busta paga... o addirittura:

fantapolitica (fantasia + politica)

ricetransmettitore (ricevitore + transmettitore)

cantautore (cantante + autore)

cantafola (canta + favola)

cartolibreria (cartoleria + libreria)

calciomercato (mercati + dei calciatori)

lavasecco (lavaggio $t-$ a secco)...

Le necessità della comunicazione tecnica richiedono un linguaggio le cui caratteristiche, oltre alla specificità del lessico, sono le costruzioni con molti nomi e pochi verbi, spesso, sequenze di puri nomi. L'eliminazione della preposizione o di altri elementi e quindi i nomi accostati direttamente, costituiscono un blocco nuovo e rendono più compatta l'espres. sione:

macchine movimento terra $=$ macchine per lavori di movimento della terra;

reparto confezioni uomo inverno $=$ reparto delle confezioni per uomo adatte nella stagione invernale;

Queste costruzioni di carattere tecnico sono frequentemente accolte nei titoli dei giornali, venendo incontro alla soluzione dello spazio limitato ed alle espressioni a effetto:

'Euroinvestivacanze', 'Vinincontri', ecc.

Alcune espressioni tecniche passano nella lingua comune e vengono usate in senso metaforico. Dal linguaggio automobilistico sono entrate nella lingua comune espressioni come: 'ingranare nel lavoro' (da 'ingranare le marce'); 'fare il pieno di ideè (da 'fare il pieno di benzina'); 'dare una sterzata', cioè cambiare bruscamente direzione, ecc. Dal linguaggio medico viene un 'espressione come: 'dare un colpo di bisturi alle spese', ossia 'tagliare decisamente, eliminare alcune spese'.

Ai linguaggio geometrico ricorrono soprattutto i politici e i giornalisti:

'una riunione triangolare ad ampio raggio';

'la quantizzazione delle tariffe'; 'il mosaico d'interventi';

'la mappatura dei rischi'...

Parimenti, fanno essi uso e abuso dei verbi del linguaggio delle scienze fisiche, tali come: elettrizzare, fossilizzare, cristallizzare, polarizzare.

Questa tendenza è, a volte, spropositata, come lo dimostrano testi veri raccolti in diverse circostanze: 
'diagnosticato un collasso della lira'; 'una terapia d'urto che freni l'emorragia di valuta'; 'occorre una radiografia della piccola industria'; 'continua l'emorragia dei capitali all 'estero'; la valenza epidemiologica ríconduce a sintesi la puntuale corrispondenza fra obiettivi e risorse'; 'il metodo partecipativo auspica un corretto rapporto fra strutture e sovrastrutture senza precostituzione delle risposte, attivando ed implementando la democratizzazione del linguaggio'.

Non manca però il 'linguaggio della vasellina'. Non potendo migliorare le condizioni economiche, scrive Marchi", si è cercato di nobilitare quelle lessicali, cosi lo 'spazzino' è diventato 'netturbino', l'infermiere' - 'paramedico', la d'omestica' - 'collaboratrice familiare', il 'facchino' — 'porta. bagagli', il 'secondino' - 'agente di custodia'. Persino la 'disoccupazione' si è attenuata in 'manodopera disponibile'.

In Italia è oggi vivo uno schema como $\mathrm{x}$ - izzare con il valore di 'Rendere simile a $\mathrm{x}$, fare di qualcosa un $\mathrm{x}$ ' : assolutizzare, atomizzare, centralizzare, estremizzare, ipotizzare, puntualizzare, ecc.

Un tempo nessuno avrebbe osato fare il superlativo di un sostantivo, e oggi si ha invece il 'campionissimo', la 'partitissima', la 'canzonissima'...

Sono stati fatti dei cataloghi abbastanza esaurienti di stilemi della pubblicità. Così sono stati identificati gli elativi come: super-, extra-, ultra- (superviscoso, extrabianco, ultrarapida); l'uso avverbiale dell,aggettivo (fissa morbido, bevi fresco, vesti giovane, mangia snello); l'uso aggettivale dell'avverbio (Crodino è più più. Una pelle così).

La publicità ricorre alle sinestesie o mescolanze di sensazioni: 'un sapore alto', 'un gusto morbido', c'ome pure alla ripetizione di serie ternarie di parole unite per asindeto: 'volante freno acceleratore-facile svelto riposante!'.

Nella comunicazione orale e scritta, vige il costrutto sintattico della duplicazione di parole che si è mantenuto vivo attraverso i secoli. Il sintagma 'via via' che anticamente significava 'subito subito', come dal dantesco: 'Per lo serpente che verrà via via' (Purgatorio VIII, 38), ha un forte riscontro nella lingua d'oggi, alla pari di 'man mano' (preferito al 'mano a mano'). Il Migliorini ${ }^{\circ}$ coniando un termine parallelo a 'fonosimbolico', ritiene che spesso si possa ricavare il valore 'morfosimbolico' della duplicazione o della triplicazione. Nell'espressione: 'rispondendo quasi colpo colpo' si può fare una sostituzione con la locuzione avverbiale 'colpo per colpo'.

4 MarChi, Cesare. Impariano l'italiano. Mllano, Rlzzoll, 1984, p.88.

5 MICLIORINI, Bruno. Lingua d’oggi e di ieri. Roma, Bclascla, 1973. p.318. 
Il costrutto, però, con un duplice complemento oggetto mostra, secondo Migliorini, morfosimbolicamente, il rapido susseguirsi dei colpi.

Strettamente affini sono le coppie: 'passo passo', 'scarpa scarpa', 'filo filo', 'pelo pelo', ossia, duplicazione di sostantivi, connessi o no con un verbo come 'andare, camminare' e simili, i quali indicano non il luogo nel quale si va, come ad esempio 'camminare riva riva', ma il modo di procedere.

Una parola che una volta apparteneva ad una specifica lingua di gruppo, ora fa parte solamente della lingua di tutti, o viceversa. Massaio era un termine dell'economia che indicava una specie di amministratore, mentre oggi, nella sua forma femminile massaia, è diventato termine comune, indica la donna in quanto opera nell'ambito dell'economia domestica.

Giacca, oggigiorno parola comune che indica indumento maschile o femminile, indicava anticamente, nel linguaggio militare, una maglia di ferro che si portava per proteggere il petto e le braccia.

Quattrino, in origine era una piccola moneta di rame, in uso dal XIII al XIV secolo, del valore di quattro denari, ha finito per indicare moneta di valore minimo, specialmente in locuzioni familiari: 'Non ho più un quattrino', 'Sono squattrinato'. Nel senso figurato, il quattrino è cosa di pochissimo valore: 'Non vale un quattrino!', 0 anche, il denaro idoleggiato: 'Il dio quattrino'. Al plurale, 'quattrini', ha l'uso comune di denaro: 'un sacco di quattrini', 'quattrini a palate', ecc.

Fasullo, derivato dalla malavita romana a cui è pervenuto dal gergo giudeo-romanesco. La parola originaria è l'ebraico post-biblico p'âsul', o 'pasul'o, cioé 'illegittimo, non valido' che si riferiva ad animali non macellati secondo il rito prescritto. In questi ultimi tempi il vocabolo si è decisa. mente imposto nella lingua parlata ed ha fatto fortuna grazie alla divulgazione da parte di giornali umoristici, radio e cinema. Voleva dire propriamente 'falso' dapprima riferendosi alle monete ed ai metalli preziosi, ma ora copre le aree semantiche di 'incapace, inservibile, sciocco, vuoto, senza senso'.

La tendenza all 'economia linguistica è testimoniata dal fenomeno dell' ellissi. Nonostante la forma ellittica registri elevato grado di accettabilità nel colloquiale, la lingua scritta, a sua volta, specialmente nella narrativa, ne fa ampio uso al focalizzare l'attenzione sulla incisività. Forme ellittiche garantiscono una piena informazione, che è immediata e ca- 
rica di effetti, come il seguente esempio, dalla esasperata sequenza di forme ellittiche congiuntamente alla paratassi: "Poi il silenzio, qualche anno muto, fino alle parole: sono stanca Marcello!"”.

La lingua italianci odierna è poco disposta verso il rigidismo grammaticale ed è avvertito nei parlanti l'uso sempre più frequente di elementi espressivi e tonali che hanno 'energia' semantica. La lingua scritta tende sempre più al parlato e supplisce, per raggiungere l'efficacia espressiva affidatta all'impostazione fonica, artifici grafici: "La Storia, è una commedia loro, che ha da finí! NOI gliela famo finí! $\mathbf{E}$ la matematica... Lo sai qual è il numero che più mi piace a'mà? 亡 lo ZERO!..."'.

Il linguaggio dei mass-media, per le incidenze che ha sulla lingua, ha notevolmente contribuito alla forrnazione dei nuovi tipi di ellissi che non investono solamente l'omissione del soggetto o del predicato, ma realizzano nuovi moduli significativi. In verità possiamo considerare l'ellissi fenomeno normale dell'italiano contemporaneo: si cerca di raggiungere la significazione evitando ogni dispendio di energie e tralasciando quegli elementi linguistici che non sono essenziali alla puntuale comunicazione. Dobbiamo ammettere, però, che in questo direzionamento della strutturazione dei messaggi la lingua tende ad automatizzarsi come un meccanismo a gettone. Ed ecco frasi a gettone: s'infila 'dialogo' ed esce 'costruttivo'; s'infila 'colloquio' ed esce 'franco e cordiale'; 'confronto' e vien fuori 'aperto'; 'intervento' e vien fuori 'tempestivo".

L'argomento sull'ellissi, posto nell'ambito situazionale del parlante, ci fa riflettere sulla dinamica delle strutture linguistiche e sul comporsi degli elementi della funzionalita del discorso. Nella costruzione della frase, il prodotto migliore dell'ellissi può senz'altro essere considerata la paratassi. Negli ultimi decenni si è ridotta la frequenza dei rapporti di subordinazione fra le proposizioni, registrandosi una progressiva evoluzione dall'ipotassi alla paratassi. Scrive il Migliorini10 che era particolarmente diffusa presso gli scrittori italiani dei primi secoli, la paraipotassi, procedimento sintattico in cui la subordinazione (ipotassi) è modificata da un elemento proprio della coordinazione (paratassi). Quasi sempre, la ripresa con e o si: " $\mathrm{E}$ cacciando in tale maniera dall'ora di prima infino all'ora di vespero, e allora per-

7 BEVILACQUA, Alberto. L'occhio del gatto. Milano, Rizzoll, 1978. p.200.

8 MORANTE. Elsa. La storia. Torino, Elnaudi, 1974. p.17g.

9 MARCHI, D.160.

10 MIGLIORINI, Bruno. Storia della lingua italiana. Firenze, Bansonl, 1962. p.408. 
venne a una fontana" (Trist, Ricc.). "E se mettea filo alle tre volte nell'ago, sì li rendeano l'arnese suo e donavanli di belli gioielli" (Novellino).

La paraipotassi è stata riscoperta ed attualizzata dagli scrittori moderni, soprattutto i naturalisti ed i veristi, spinti dallo zelo ad una descrizione documentaria della realtà, ed è tuttora ampiamente usata.

Nell'individuare le cause del progressivo evolversi della lingua dalla ipotassi alla paratassi, è stato rilevato che il linguaggio assume sempre più una funzione pragmatica per cui la sua dimensione simbolica tende a ridursi a quella dimostrativa. Risulta chiaro che laddove prevale l'immediatezza della comunicazione, come nella lingua parlata, l'utente ricorre alla più spontanea operazione della paratassi, mentre seleziona strutture ipotattiche per le esigenze del pensiero riflesso.

Dopo quanto constatato, è pertinente una domanda: "Esiste o non esiste il congiuntivo nell'italiano odierno? $\mathrm{E}$, se esiste, in che misura é una semplice variante dell'indicativo o invece un modo obbligatorio e autonomo?". In vista della nostra perspettiva puramente sincronica, dobbiamo ammettere che sono considerati nell'italiano contemporaneo i tempi del congiuntivo: il presente ed il passato, trascurati invece l'imperfetto ed il trapassato. Il congiuntivo è più che altro il portatore di una informazione di persona e di aspetto, ma non possiede valore temporale. Facciamo un esempio:

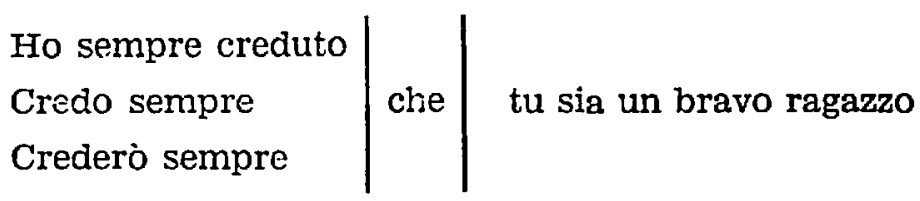

Qualunque sia il valore deitico temporale del processo. il congiuntivo non cambia. L'indicativo è il solo modo dell'immediatezza e della contemporaneità, intese nel rapporto enunciato/enunciazione. I tempi passati dell'indicativo indicano l'anteriorità rispetto al momento dell'enunciazione, ed il futuro, la posteriorità a questo momento. Ciò costituisce una delle ragioni per cui il trapassato remoto si usa esclusivamente nello scrivere, e per giunta, nello scrivere di registro aulico. Il passato prossimo, invece, è incontrato tanto nella scritta quanto nel parlato. Anche il futuro anteriore è tipico della lingua scritta, nel parlare, generalmente, si sostituisce con forme più semplici: "Sarà stato Giovanni a fumare" $\rightarrow$ "Forse è stato Giovanni a fumare". 
Quanto al condizionale, tre sono le funzioni più ricorrenti:

1. il condizionale giornalistico, la cui funzione consiste nel segnalare che la notizia non sembra provenire da fonte sicura;

2. il condizionale con funzione prospettiva, usato nella narrativa allorché il narratore vuole anticipare un evento senza ricorrere al vero futuro (il che implicherebbe l'intervento soggettivo del narratore);

3. il condizionale di modestia. Quest'ultimo caso ha delle implicazioni sociologiche e psicologiche, specialmente trattandosi di condizionale inserzionistico. A riprova, c'è il fatto che negli annunci economici, le formule più frequenti sono: assumesi/assumonsi e assumerebbe/accetterebbe. Chiunque è nelle condizioni di poter scegliere, adopera l'indicativo, a cominciare dai datori di lavoro: "offriamo interessanti guadagni", "selezioniamo personale", "assumiamo operai qualificati". Viceversa, nella rubrica delle domande di lavoro prevale statisticamente il condizionale: 'giovane laureata impartirebbe lezioni di matematica", "pensionato assumerebbe servizio di portineria". Molto diffusa è la formula "offresi", ma il verbo stesso, nonostante all'indicativo, esprime umiltà.

La frequenza di utilizzazione del gerundio segue una curva decrescente dall'italiano antico all'italiano odierno; dai registri alti, formali, ai registri medi. Nell'ambito di questa tendenza si collocano anche le varie alternative di realizzazione del gerundio da un punto di vista semanticosintattico: l'italiano aulico odierno presenta il ventaglio più ampio di varietà semantico-sintattiche. Al contrario, nei campioni medi, sono rappresentate pochissime varianti delle quali la più ricorrente è quella del tipo: stare + infinito. Per quanto riguarda la categoria temporale, il gerundio passato tende a sparire. Per l'italiano delle origini Segre ${ }^{11}$ attribuisce al gerundio una funzione narrativa per cui, specialmente come viene impiegato da Guittone, indica molto superficialmente i legami logici di subordinazione, sottolineando invece il puro fatto della subordinazione in sé stante.

Oggi il carattere di subordinatore sopravvive nel tipo ormai più frequente, il GRMSV, cioè il gerundio modificatore di sintagma verbale che raggiunge approssimativamente il $70 \%$ nell'italiano corrente ed il $100 \%$ nella pubblicità.

11 SEGRE, Cesare. Lngua, stilo o socicta. Mtlano. Feltrinelli, 1974, p.123. 


\section{RESUMO}

Trata-se de um esboço da situação atual da lingua italiana cuja análise consistiu em testar amostras representativas da língua escrita e falada, com especial atençāo ì linguagem jornalistica e publicitária.

\section{REFERENCIAS BIBLIOGRAFICAS}

1 BEVILACQUA, Alberto. L'occhia del gatto. Milano, Rizzoll, 1978. $260 \mathrm{p}$.

2 DEVOTO, Giacomo. Civiltá di parole. Firenze, Sansoni, 1965. 297 p.

3 DIATTO \& MORTARA. Messaggio e comunicazione. Torino, Loescher, 1977. 392 p.

4 IADAROLA, Mario. Mondo parola. Torino, Lattes, 1982. 680 p.

5 MARCHI, Cesare. Impariamo l'italiano. Milano, Rizzoli, 1984. 177. p.

6 MIGLIORINI, Bruno. Lingua d'oggi e di ieri. Roma, Sciascia, 1973.

7 Storia della lingua italiana. Firenze, Sansoni, 1962. $708 \mathrm{p}$.

8 MORANTE, Francesco. Pratica, analisi e storia della lingua italiana. Torino, Lattes, 1981. $730 \mathrm{p}$.

10 SEGRE, Cesare. Lingua, stile e societa. Milano, Feltrinelli, 1974. 237 p. 